\title{
Effect of the local environment on the mobility of dislocations in refractory bcc metals: Concurrent multiscale approach
}

\author{
Zhengzheng Chen, ${ }^{1}$ Gang Lu, ${ }^{1}$ Nicholas Kioussis,${ }^{1}$ and Nasr Ghoniem ${ }^{2}$ \\ ${ }^{1}$ Department of Physics, California State University, Northridge, California 91330-8268, USA \\ ${ }^{2}$ Department of Mechanical and Aerospace Engineering, University of California-Los Angeles, Los Angeles, California 90095, USA
}

(Received 16 July 2008; published 6 October 2008)

\begin{abstract}
Using a concurrent multiscale approach we demonstrate that the local environment of transition-metal solutes in refractory bcc metals has a large effect on the mobility and slip paths of dislocation. The results reveal that solid solutes or nanoclusters of different geometries may lead to solid-solution hardening or softening, in agreement with experiment, including spontaneous dislocation glide and activation of new slip planes. The underlying electronic mechanism is the change in the anisotropy of the lattice resistance induced by solutes.
\end{abstract}

DOI: 10.1103/PhysRevB.78.134102

PACS number(s): 61.72.Lk, 71.15. $-\mathrm{m}, 62.20 . \mathrm{F}-$

Solute atoms are ubiquitous in metals and play a key role in altering their mechanical properties (e.g., strength and ductility). Experimental studies during the past several decades indicate that solutes can give rise to both solid-solution hardening $(\mathrm{SSH})$ and solid-solution softening (SSS). ${ }^{1,2}$ The origin of SSH/SSS is due to the interaction between dislocations and solutes and/or precipitates in materials. In most situations, the dislocation behavior is considerably different in the realistically dirty materials, where dislocation mobility can vary by several orders of magnitude. This significant influence induced by small amounts of solutes is of great practical importance to, for example, the bcc refractory metals ( $\mathrm{Nb}, \mathrm{W}, \mathrm{Ta}$, and $\mathrm{Mo}$ ). These systems exhibit unique mechanical properties that make them attractive for structural applications at elevated temperatures. An inherent drawback limiting the use of these materials as structural components is their reduced low-temperature toughness, which in turn increases the propensity toward fracture. Thus, the challenge in design of advanced alloys is to combine strengthening and toughening phases with a better balance of properties.

Continuum elasticity theory has provided considerable insight of SSS/SSH in terms of the size and elastic constants between the solute and host atoms. The correlation between the hardening rate and number of conduction electrons of transition-metal solutes, however, indicates a nonlinear chemical origin of the dislocation-solute interaction. ${ }^{3,4}$ In their pioneering work, Trinkle and Woodward ${ }^{3}$ using the first-principles Greens function boundary condition (FPGFBC) method, ${ }^{5}$ demonstrated the transition-metal solutes can have a large effect on the dislocation core and hence the mobility. Nevertheless, understanding the physics of interactions of dislocations with nanoclusters remains a challenging problem.

In this paper, we have developed a concurrent multiscale approach, which opens the door to studying the important problem of chemistry effect on the mechanical properties of metals. This approach treats correctly the long-range elastic field of the dislocation and describes the solute-host atomic interaction in the core region accurately. We have applied this approach to the Ta-W alloys, two prototype bcc metals, because (1) experiments have shown the dual nature of $\mathrm{W}$ : the addition of $2.5-10.0 \mathrm{wt} . \% \mathrm{~W}$ in Ta increases the strength while it enhances substantially the ductility at high strain-rates; ${ }^{6,7}$ and (2) the high solubility of $\mathrm{W}$ in Ta allows a wide range of local environments. The results demonstrate the multinature of $\mathrm{W}$ solutes. Depending on the solute local environment (random solid solution or nanoclusters of different geometry), the mobility may exhibit a wide spectrum of unusual behavior: SSS, SSH, or even a spontaneous dislocation glide in the absence of external stress. Finally, W solutes can serve as obstacles to dislocation motion and activate new slip planes.

The concurrent multiscale approach is based on that proposed by Choly et al. ${ }^{8}$ The system, shown in Fig. 1, is divided into: region $\mathrm{I}$, which includes the dislocation core and the solutes, is treated within the framework of densityfunctional theory (DFT); region II which refers to the rest of the system is treated with the embedded atom method (EAM) or Finnis-Sinclair (FS) potentials; and the boundary (B) region introduced to reduce the coupling errors between

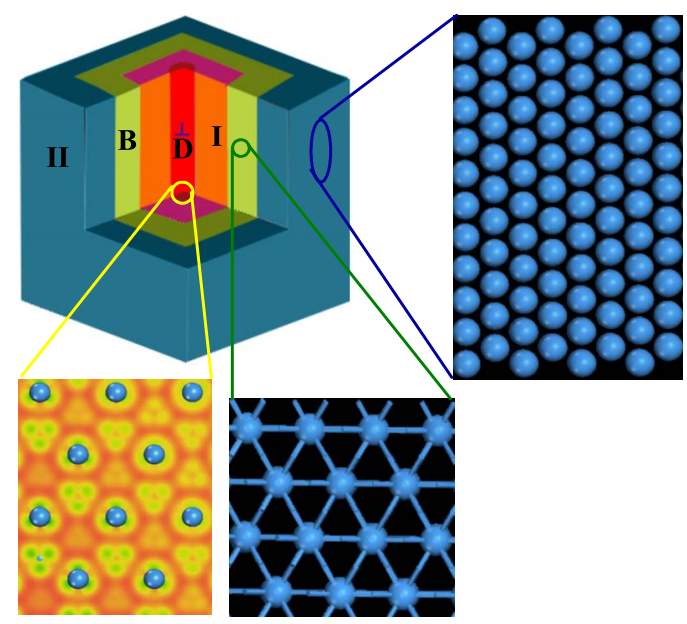

FIG. 1. (Color online) Schematic view of the partitioned multiscale system: Region I (red/gray) contains the core of the screw dislocation (marked by D); Region II (blue/dark gray) consists of atoms treated with EAM; and the boundary $(\mathbf{B})$ region (green/light gray) consists of atoms on the boundary between regions I and II. Periodic boundary conditions are used along the $\langle 111\rangle$ dislocation line. 
regions I and II, respectively. The total energy of the system can be written as ${ }^{8}$

$$
E[\mathrm{I}+\mathrm{II}]=E_{\mathrm{DFT}}[\mathrm{I}]+E_{\mathrm{EAM}}[\mathrm{II}]+E^{\mathrm{int}}[\mathrm{I}, \mathrm{II}] .
$$

Here, $E_{\mathrm{DFT}}[\mathrm{I}]$ is the energy of region $\mathrm{I}$ in the absence of region II obtained by DFT calculations, $E_{\mathrm{EAM}}[\mathrm{II}]$ is the energy of region II obtained by EAM calculations in the absence of region I, and $E^{\mathrm{int}}[\mathrm{I}, \mathrm{II}]$ represents the interaction energy added to give the correct total energy of the whole system. In the present approach $E^{\mathrm{int}}[\mathrm{I}, \mathrm{II}]$ is calculated by the EAM potential,

$$
E^{\mathrm{int}}[\mathrm{I}, \mathrm{II}]=E_{\mathrm{EAM}}[\mathrm{I}+\mathrm{II}]-E_{\mathrm{EAM}}[\mathrm{I}]-E_{\mathrm{EAM}}[\mathrm{II}] .
$$

This treatment of the interaction energy yields the following expression for the total energy:

$$
E[\mathrm{I}+\mathrm{II}]=E_{\mathrm{EAM}}[\mathrm{I}+\mathrm{II}]-E_{\mathrm{EAM}}[\mathrm{I}]+E_{\mathrm{DFT}}[\mathrm{I}],
$$

where $E_{\mathrm{EAM}}[\mathrm{I}+\mathrm{II}]$ is the energy of the entire region calculated from the EAM, and $E_{\mathrm{EAM}}[\mathrm{I}]$ and $E_{\mathrm{DFT}}[\mathrm{I}]$ are the energies of region I in the absence of region II obtained by EAM and DFT calculations, respectively.

Differentiation of Eq. (3) with respect to the atomic positions $\mathbf{R}^{\mathrm{I}}$ and $\mathbf{R}^{\mathrm{II}}$ in regions I and II, respectively, yields that the forces on the DFT and EAM atoms are

$$
\left\{\begin{array}{l}
F_{i}[\mathrm{I}]=-\frac{\partial E[\mathrm{I}+\mathrm{II}]}{\partial R_{i}^{\mathrm{I}}}=F_{i}^{\mathrm{EAM}}[\mathrm{I}+\mathrm{II}]-F_{i}^{\mathrm{EAM}}[\mathrm{I}]+F_{i}^{\mathrm{DFT}}[\mathrm{I}], \\
F_{i}[\mathrm{II}]=-\frac{\partial E[\mathrm{I}+\mathrm{II}]}{\partial R_{i}^{\mathrm{II}}}=F_{i}^{\mathrm{EAM}}[\mathrm{I}+\mathrm{II}] .
\end{array}\right.
$$

Equation (4) shows that the forces on the atoms in region II are solely determined by the EAM potential while those on the atoms in region I have several contributions. The boundary between regions I and II introduces a fictitious surface, which in turn gives rise to substantial ghost forces on the atoms which lie the vicinity of the boundary, namely, in region $\mathbf{B}$. These forces, which are due to the mismatch in the DFT and EAM forces on the surface atoms and the fictitious surface, severely degrade the accuracy of the approach. In order to improve the quality of the coupling across the boundary we have proposed an improved coupling scheme in our previous work. ${ }^{9}$ The correction force on the atoms in $\mathbf{B}$ is

$$
F_{i}^{\text {corr }}[\mathbf{B}]=-F_{i}^{\mathrm{DFT}}[\mathbf{B}]+F_{i}^{\mathrm{EAM}}[\mathbf{B}],
$$

yielding

$$
F_{i}[\mathbf{B}]=F_{i}^{\mathrm{EAM}}[\mathrm{I}+\mathrm{II}]
$$

for the force on these atoms. The introduction of the correction force requires the corresponding correction of the total energy $E_{\text {tot }}$ of the entire system to guarantee the consistency between the forces and the energy. Thus, the total energy $E_{\text {tot }}$ becomes

$$
E_{\mathrm{tot}}=E[\mathrm{I}+\mathrm{II}]-\sum_{i \in \mathbf{B}} F_{i}^{\mathrm{corr}}[\mathbf{B}] \cdot u_{i}[\mathbf{B}],
$$

where $u_{i}[\mathbf{B}]$ is the displacement of a boundary atom at each relaxation step and the sum is over all atoms in $\mathbf{B}$. $E_{\text {tot }}$ is

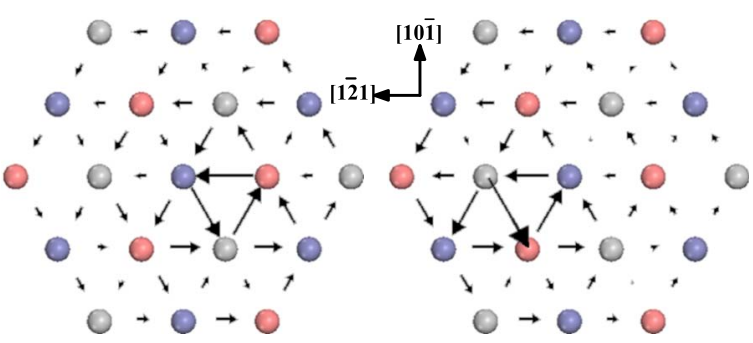

FIG. 2. (Color online) DD map of the core of the screw dislocation in Ta under (a) zero stress (left panel) and (b) $1.8 \mathrm{GPa}$ (right panel). Circles of different color (brightness) represent atoms on three successive (111) planes.

minimized by employing conjugate gradient techniques until the maximum force is smaller than $0.01 \mathrm{eV} / \AA$. More details of the methodology can be found in Ref. 9.

The size of the entire system is $251 \AA \times 145 \AA$ $\times 2.86 \AA$ along the $\langle 110\rangle,\langle 112\rangle$, and $\langle 111\rangle$ directions, respectively, containing 5400 atoms. Region I is $22 \AA$ $\times 28 \AA \times 2.86 \AA$ and contains 96 atoms. All atoms are initially displaced according to the isotropic elastic solution of the screw dislocation with Burgers vector $\vec{b}=\langle 111\rangle a / 2$, and are then relaxed by the concurrent multiscale approach, which takes into account the anisotropic effects. Periodic boundary conditions are applied along the $\langle 111\rangle$ direction and fixed boundary conditions are employed along the other two directions, respectively. The DFT cluster calculations in region I were carried out using the VASP code. ${ }^{10,11}$ We have used a $1 \times 1 \times 8 k$ mesh according to the Monkhorst-Pack scheme. $^{12}$ The generalized gradient approximation functiona ${ }^{13}$ is used to treat the exchange and correlation potential, and the cutoff energies are set at $240 \mathrm{eV}$ for both Ta and W. The EAM calculations employed the Ackland potential $^{14}$ for pure Ta and the Johnson scheme ${ }^{15}$ for constructing the Ta-W interatomic potential. It should be emphasized that the quality of the Ta-W classical interaction is not crucial in this multiscale approach, due to the cancelation between the first two energy terms in Eq. (3), provided that the various atomic species are well within region I. The external stress is applied by displacing equally the atoms in the outermost $(10 \overline{1})$ and (101) planes along the [ $\overline{1} \overline{1} \overline{1}]$ and [111] directions, respectively. The corresponding shear stress, $\sigma_{y z}$, is calculated as the component of the area-averaged force on the surfaces parallel to the Burgers vector.

In Fig. 2 we show the relaxed dislocation core structure in pure Ta under zero stress (left panel) and $1.8 \mathrm{GPa}$ (right panel), respectively, using the differential displacement (DD) maps. ${ }^{16}$ The arrows indicate the relative $\langle 111\rangle$ displacement of neighboring atoms of the dislocation. The length (direction) of the arrow denotes the magnitude (sign) of the displacement difference. When the arrow touches the centers of the two atoms, their relative displacement is $b / 3$. The dislocation core for pure Ta is nondegenerate and spreads symmetrically on the six planes. Upon increasing $\sigma_{y z}$ on the $(10 \overline{1})$ planes, the dislocation center moves to the next Peierls valley at the critical stress $\sigma_{P}$ of $1.8 \mathrm{GPa}$. Comparison of $\sigma_{P}$ and the core structure in Table I employing different approaches demonstrates that the concurrent multiscale 
TABLE I. Peierls stress $\left(\sigma_{P}\right)$ and core structure for a screw dislocation in Ta using the present multiscale approach, the FPGFBC method, the EAM, and the modified generalized pseudopotential theory (MGPT).

\begin{tabular}{lcc}
\hline \hline & $\sigma_{P}(\mathrm{GPa})$ & Core structure \\
\hline present work & 1.8 & Nondegenerate \\
FP-GFBC $^{5}$ & 1.8 & Nondegenerate \\
$\mathrm{EAM}^{17}$ & 1.8 & Degenerate \\
$\mathrm{MGPT}^{18}$ & 0.6 & Nondegenerate \\
\hline \hline
\end{tabular}

approach is in excellent agreement with the results of the FP-GFBC method. ${ }^{5}$

In order to show the effect of the fixed boundary condition when the dislocation moves, we plot in Fig. 3 the atomic displacement for pure Ta along the $\langle 111\rangle$ direction normalized to $b$ for all atoms lying in a circular shell of $30 \AA$ radius centered at the dislocation core versus the angle $\theta$ with respect to the $[\overline{1} \overline{1} \overline{1}]$ direction. Note that the dislocation core has moved by $2.7 \AA$ along the [1 121$]$ direction under the stress of $1.8 \mathrm{GPa}$. Thus, the dislocation core is still quite far $(\approx 115 \AA)$ from the boundary of the entire system consisting of regions I and II. The displacement fields for $0 \mathrm{GPa}$ and 1.8 $\mathrm{GPa}$, respectively, are very similar. The maximum difference in atomic displacement is about $2 \%$ of $b$. Thus, these results demonstrate that the boundary condition does not have an effect on the dislocation core structure and the Peierls stress.

In order to study the effect of local environment of the solute on the mobility of the screw dislocation, we have considered the cases of (i) dilute random solid solutions where the dislocation line is affected only by isolated solute atoms (ISA) shown in Fig. 4(a), and (ii) small clusters of solute atoms of hexagonal shape (CSA-H) shown in Fig. 4(b) or of triangular shape (CSA-T) shown in Fig. 4(c). In all cases the $\mathrm{W}$ concentration is $\sim 6$ at $\%$ and the $\mathrm{W}$ atoms are denoted with black circles. The upward triangle, downward triangle, square, and solid dot denote the position of the dislocation

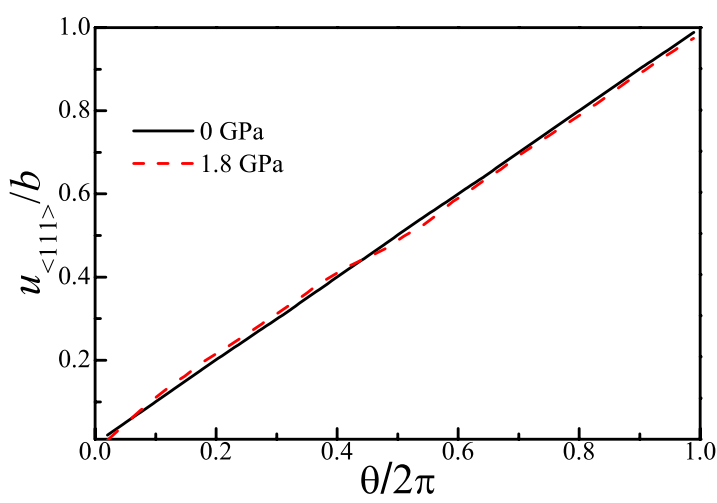

FIG. 3. (Color online) Atomic displacement for pure Ta along $\langle 111\rangle$ normalized to $b$ for all atoms lying in a circular shell of $30 \AA$ radius centered at the dislocation core versus the angle $\theta$ with respect to the $[\overline{1} 2 \overline{1}]$ direction. The black solid and red (gray) dashed curves correspond to 0 and $1.8 \mathrm{GPa}$ external shear stress, respectively.

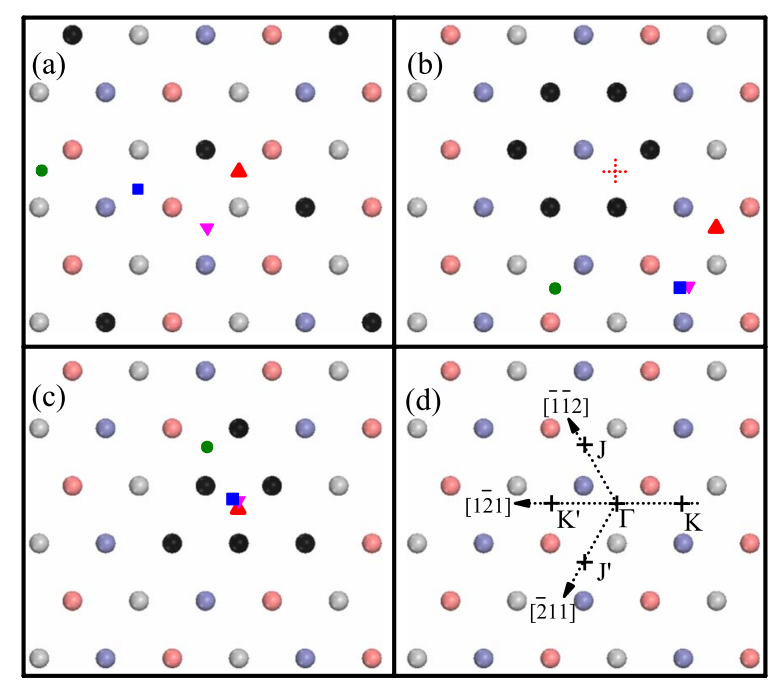

FIG. 4. (Color online) Screw dislocation slip paths under stress for three configurations of $\mathrm{W}$ solutes denoted by black circles: (a) isolated solute atoms (ISA) and nanoclusters of (b) hexagonal (CSA-H) or (c) triangular (CSA-T) shape. The upward triangle, downward triangle, square, and solid dot denote the position of the core center under $0.0,1.0,1.5$, and $2.0 \mathrm{GPa}$, respectively. Panel (d) displays the special points and directions in Fig. 5. $\Gamma$ indicates the initial position of the dislocation.

center under $0.0,1.0,1.5$, and $2.0 \mathrm{GPa}$, respectively. In the absence of stress the core center is at point $\Gamma$ in panel (d) for the ISA and the CSA-T cases, while it is unstable [dashed cross in panel (b)] for the CSA-H case. Interestingly, in the latter case the dislocation center is repelled by the CSA-H and spontaneously moves to the position of the upward triangle under the precipitate-induced chemical stress. Under 1.0 $\mathrm{GPa}$, both in the ISA and CSA-H cases the dislocation prefers to bypass the $\mathrm{W}$ sites and moves on the $(0 \overline{1} 1)$ plane. Upon increasing the stress to $1.5 \mathrm{GPa}$, the behavior changes: the dislocation in ISA continues to move, while it is pinned in CSA-H. The dislocation in CSA-H begins to move further under $1.8 \mathrm{GPa}$, as in the case in pure Ta. In sharp contrast to the other two cases, the dislocation core is pinned at $\Gamma$ in the CSA-T case until the stress reaches $2.0 \mathrm{GPa}$, where the center moves on the (110) glide plane. The calculated values of critical stress, $\sigma_{P}$, and polarization, ${ }^{17} p$, are listed in Table II, for the ISA, CSA-H, and CSA-T environments. Both ISA and CSA-H result in SSS, while CSA-T produces SSH. In all cases, W solutes have a small effect on the polarization, indicating the absence of correlation between the polarization and $\sigma_{P}$.

Edagawa et al. ${ }^{19}$ suggested that the dislocation motion in

TABLE II. $\sigma_{P}(\mathrm{GPa})$ and polarization (Ref. 17), $p$, for isolated solute atoms (ISA), nanoclusters of solute atoms of hexagonal shape (CSA-H), or of triangular shape (CSA-T), shown in Fig. 4.

\begin{tabular}{lccc}
\hline \hline & ISA & CSA-H & CSA-T \\
\hline$\sigma_{P}$ & 1.0 & 1.0 & 2.0 \\
$p$ & 0.10 & 0.04 & 0.00 \\
\hline
\end{tabular}



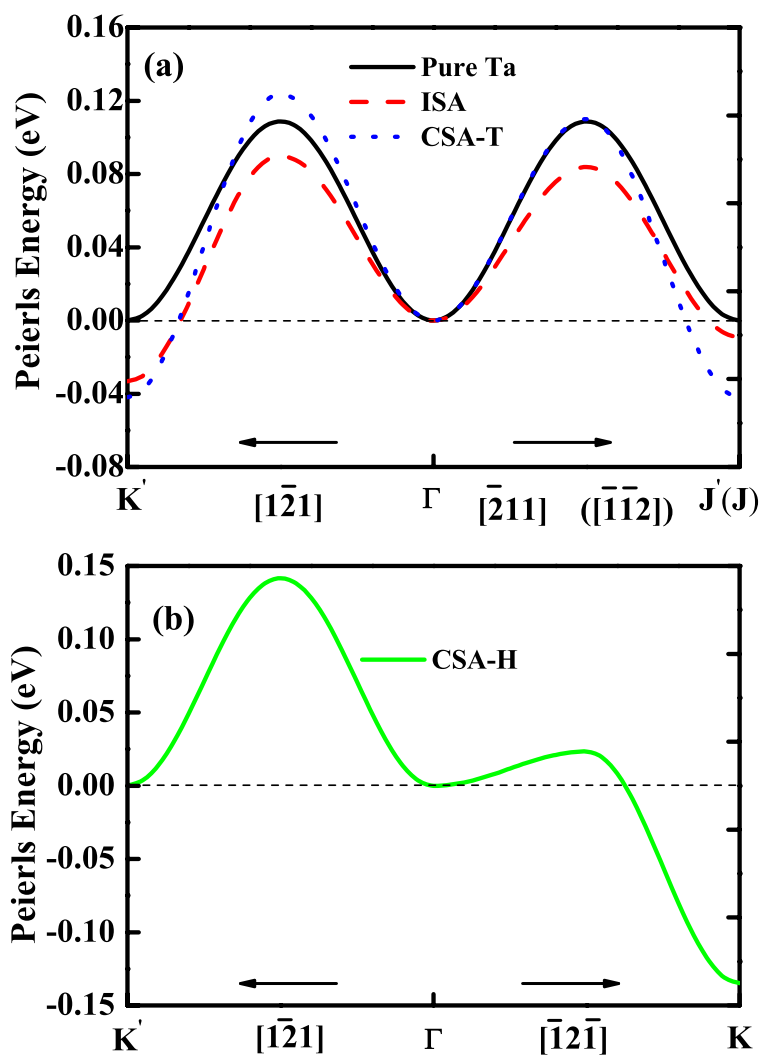

FIG. 5. (Color online) Peierls potential along the various $\langle 1 \overline{2} 1\rangle$ directions in Fig. 4(d) for (a) Pure Ta (solid curve), the ISA (dashed curve), and the CSA-T (dotted curve), and (b) for the CSA-H. The direction and special point in parenthesis in Fig. 5(a) correspond to the CSA-T environment.

bcc metals can be represented by the Peierls potential surface (PPS), a two-dimensional (2D) surface perpendicular to the Burgers vector. The profile of PPS is a valuable quantity to measure the lattice resistance to dislocation motion. Using the present approach we have determined the PPS by moving the dislocation center along different directions in Fig. 4(d). The PPS along the various $\langle 1 \overline{2} 1\rangle$ directions for pure Ta, the ISA, and the CSA-T environments is shown in Fig. 5(a) and the corresponding PPS for CSA-H is shown in Fig. 5(b). The Peierls barrier along the $[1 \overline{2} 1]\left(\Gamma-K^{\prime}\right)$ and $[\overline{2} 11]\left(\Gamma-J^{\prime}\right)$ directions are reduced compared to pure Ta, consistent with the results of $\sigma_{P}$ in Table II. On the other hand, the CSA-H environment has a large effect on the PPS along the [ $\overline{1} 2 \overline{1}]$ direction, where the Peierls barrier between $\Gamma$ and $K$ is greatly reduced, which is the reason why the dislocation glides spontaneously (under zero stress) in Fig. 4(b). Finally, for the CSA-T configuration, the barrier along [121] on the (101) plane increases, while that along [ $\overline{1} \overline{1} 2]$ on the $(\overline{1} 10)$ plane remains the same as in Ta. Thus, the dislocation first

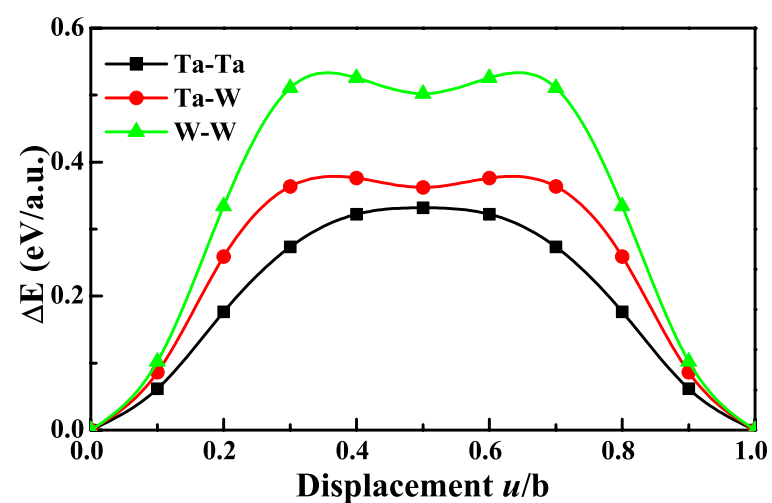

FIG. 6. (Color online) Energy change per unit length, $\Delta E^{(X-X)}\left(\frac{u}{b}\right)(X=\mathrm{Ta}, \mathrm{W})$, versus the normalized atomic-row displacement $u / b$ along $\langle 111\rangle$.

glides on the $(\overline{1} 10)$ plane, which is different from that in pure Ta.

In order to understand the underlying mechanism of the dislocation-solute interaction, we present in Fig. 6 the change in energy, $\Delta E^{(X-X)}\left(\frac{u}{b}\right)$, per unit length to displace an atomic row of $X$ atoms $(X=$ Ta, W) by $u$ along $\langle 111\rangle$ relative to a nearest-neighbor Ta atomic row. ${ }^{3,4,20}$ We find that $\mathrm{W}$ increases substantially the inter-row shear energies for the Ta-W and W-W rows and the lattice resistance when the dislocation center is in the vicinity of W. This in turn increases $\sigma_{P}$ and hence SSH. On the other hand, the dislocation tends to bypass $\mathrm{W}$ and changes the slip plane and/or slip direction. This could be achieved by either cross slip or kink formation. The definitive mechanism requires further study. The interplay between dislocation pinning, cross slip, and kink nucleation is responsible for the multitude of plastic behavior observed in solid-solution alloys.

In summary, we have employed a concurrent multiscale approach to study the effect of chemistry on the dislocation mobility in refractory bcc metals. The unique feature of this approach is that it treats correctly the long-range dislocation strain field, while allowing the quantitative treatment of the effect of chemistry on the dislocation core. The multiscale approach yields a core structure and dislocation mobility for $\mathrm{Ta}$ in excellent agreement with the FP-GFBC method. ${ }^{5} \mathrm{We}$ have demonstrated that the local environment of $\mathrm{W}$ solutes changes the anisotropy of the lattice resistance, and hence it has a large effect on both the dislocation mobility and slip paths: it may lead to SSS, SSH, or even a spontaneous dislocation glide.

We acknowledge valuable discussions with V. Vitek. This research was supported by U.S. DOE NERI under Grant No. DE-FC07-06ID14748, NSF-PREM under Grant No. DMR00116566, U.S. Army Research Office under Grant No. W911NF-04-1-0058, and U.S. DOE SciDAC under Grant No. DE-FC02-06ER25791. 
${ }^{1}$ E. Pink and R. J. Arsenault, Prog. Mater. Sci. 24, 1 (1979).

${ }^{2}$ Y. F. Gu, H. Harada, and Y. Ro, J. Met. 56, 28 (2004).

${ }^{3}$ D. R. Trinkle and C. Woodward, Science 310, 1665 (2005).

${ }^{4}$ N. I. Medvedeva, Yu. N. Gornostyrev, and A. J. Freeman, Phys. Rev. Lett. 94, 136402 (2005).

${ }^{5}$ C. Woodward and S. I. Rao, Phys. Rev. Lett. 88, 216402 (2002). ${ }^{6}$ W. H. Gourdin, D. H. Lassila, M. M. LeBlanc, and A. L. Shields, J. Phys. (Paris), Colloq. 4, C8-207 (1994).

${ }^{7}$ C. L. Briant and D. H. Lassila, J. Eng. Mater. Technol. 121, 172 (1999).

${ }^{8}$ N. Choly, G. Lu, E. Weinan, and E. Kaxiras, Phys. Rev. B 71, 094101 (2005).

${ }^{9}$ Y. Liu, G. Lu, Z. Z. Chen, and N. Kioussis, Modell. Simul. Mater. Sci. Eng. 15, 275 (2007).

${ }^{10}$ P. E. Blöchl, Phys. Rev. B 50, 17953 (1994).

${ }^{11}$ G. Kresse and J. Furthmuller, Phys. Rev. B 54, 11169 (1996).
${ }^{12}$ H. J. Monkhorst and J. D. Pack, Phys. Rev. B 13, 5188 (1976).

${ }^{13}$ J. P. Perdew, K. Burke, and M. Ernzerhof, Phys. Rev. Lett. 77, 3865 (1996).

${ }^{14}$ S. Han, L. Zepeda-Ruiz, G. Ackland, R. Car, and D. Srolovitz, J. Appl. Phys. 93, 3328 (2003); also http://homepages.ed.ac.uk/ graeme

${ }^{15}$ R. A. Johnson, Phys. Rev. B 39, 12554 (1989).

${ }^{16}$ V. Vitek, Cryst. Lattice Defects 5, 1 (1974).

${ }^{17}$ G. Wang, A. Strachan, T. Çă̆in, and W. A. Goddard III, Mater. Sci. Eng., A 309-310, 133 (2001).

${ }^{18}$ L. H. Yang, P. Söderlind, and J. A. Moriarty, Philos. Mag. A 81, 1355 (2001).

${ }^{19}$ K. Edagawa, T. Suzuki, and S. Takeuchi, Phys. Rev. B 55, 6180 (1997).

${ }^{20}$ Z. Z. Chen, N. Kioussis, N. Ghoniem, and T. Hasebe, Phys. Rev. B 77, 014103 (2008). 